\section{Superbug genome excels at passing on drug resistance}

Jim Giles, London

A bacterium that is spreading through

hospitals because of its resistance to certain antibiotics is well equipped to develop yet more self-protection, according to a study of its genome.

Methicillin-resistant Staphylococcus aureus (MRSA) kills about 800 patients a year in Britain alone, and health officials have been watching its spread with alarm (see Nature 422, 791; 2003).

A genome sequence for one of its strains, published by a group at the Wellcome Trust Sanger Institute near Cambridge, UK, is significantly different from the six other resistant and nonresistant strains that have been sequenced (M. T. G. Holden et al. Proc. Natl Acad. Sci. USA 101, 9786-9791; 2004). And the areas that differ contain the genes that confer resistance to drugs.

The finding suggests that resistance genes can transfer very quickly between different strains, says Julian Parkhill, a geneticist in the Sanger team. "This is extremely bad news," he adds. "Strains don't have to develop resistance independently."

Other researchers now fear that many strains of the bacteria could acquire resistance to the antibiotic vancomycin, which is commonly used to treat patients infected with MRSA. Few other effective treatments are available.

In May, a study led by Mark Enright, a microbiologist at the University of Bath, UK, suggested that resistance to vancomycin was developing independently in several strains of MRSA (R. A. Howe et al. Emerg. Infect. Dis. 10, 855-857; 2004).

Vancomycin-resistant strains were first identified in 1997 and had been thought rare. But Enright looked at about 100 samples of MRSA that had shown some vancomycin resistance. He found that the microbes were related to the five major MRSA strains that cause problems in hospitals, and not to a single strain as some had originally thought.

The implication, says Enright, is that resistance is evolving in strains around the world. "Outbreaks are inevitable as long as we keep using vancomycin," he says.

Researchers do not know when full vancomycin resistance will become widespread. Only three cases have been reported so far, and none led to fatalities. But Enright says that deaths from the bacteria can go unrecorded. "If people are ill anyway, you will not necessarily see MRSA on the death certificate."

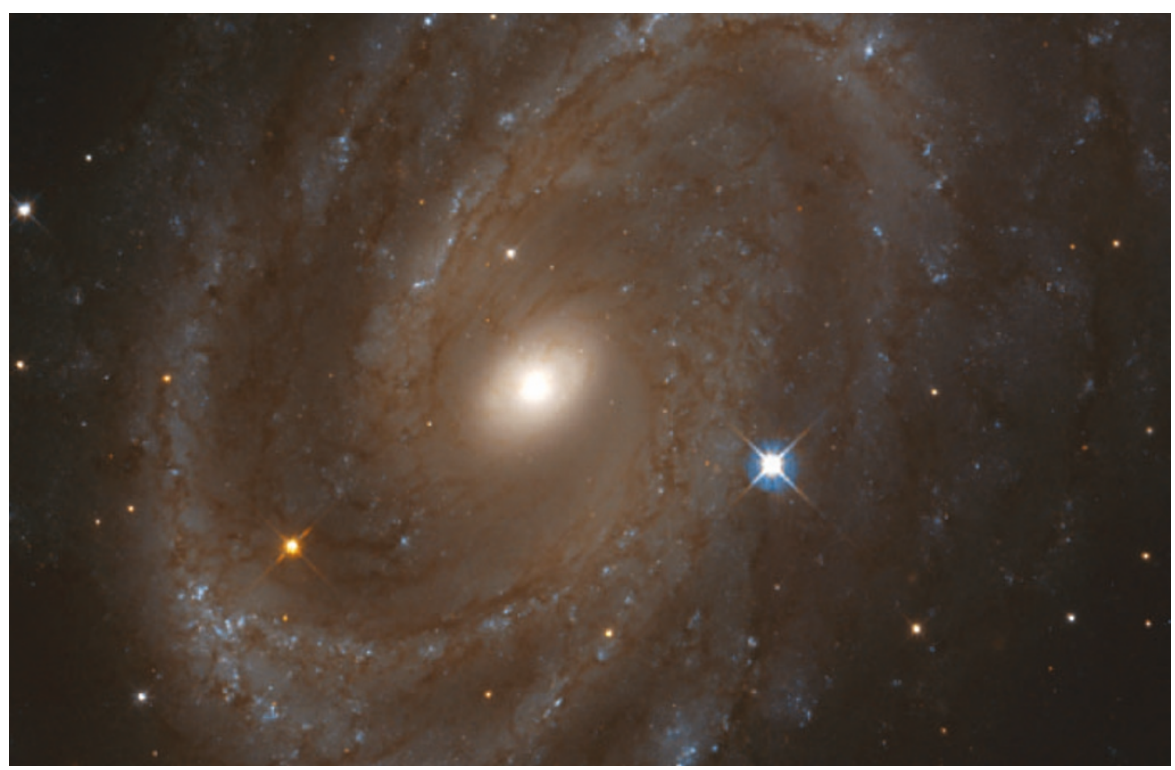

Give it a whirl: could a simple test detect energy that, so far, has only been measured by cosmologists?

\title{
Scepticism greets pitch to detect dark energy in the lab
}

Philip Ball

A cosmic force that is thought to drive the accelerating expansion of the Universe could be probed using desktop electronics, two researchers have claimed.

The force — usually known as dark energy — seems to oppose gravity, making galaxies fly apart with increasing speed. Detected eight years ago, it presents one of the biggest puzzles in cosmology.

But we may not need high-powered telescopes to study it, according to Christian Beck, a mathematical physicist at Queen Mary, University of London, and mathematical biologist Michael Mackey of McGill University in Québec (C. Beck \& M. C. Mackey, preprint at xxx.arxiv.org/abs/astro-ph/0406504; 2004).

They say that noise found in a commonplace electronic device could be the fingerprint of dark energy. If it is, they predict this noise will fall quiet above a well-defined frequency. The idea has yet to be tested.

Other researchers are sceptical, however. "The paper is intriguing," says physicist Peter Milonni of the Los Alamos National Laboratory in New Mexico, "but I think it would be a bit of a stretch to say that it holds water."

Cosmologist Robert Caldwell of Dartmouth College in Hanover, New Hampshire, applauds the idea of lab tests but says that for this one to work, "it would have to violate things we already know to be true".

Dark energy is estimated to account for nearly three-quarters of all the energy in the Universe - but no one really knows what it is or where it comes from. One possible source is a buzzing of empty space known as vacuum fluctuations. Quantum theory implies that a vacuum is constantly fizzing with particles that pop in and out of existence. Scientists assume that a natural mechanism cancels out the energy this produces, but some wonder if a little survives to give rise to dark energy.

The effects of vacuum fluctuations can show up in the lab. For example, they make two parallel plates separated by a vacuum attract one another, a phenomenon called the Casimir effect. They are also believed to account for some of the noise in Josephson junctions, devices in which an insulating layer is sandwiched between two superconductors.

If vacuum fluctuations are responsible for dark energy, say Beck and Mackey, then the energy density of these fluctuations should be the same as the density of dark energy that has been calculated from astronomical observations.

Calculating the energy density in Josephson-junction noise means adding up the energy at all frequencies. So far, measurements have been made up to almost a trillion hertz. They already give a density that is about equal to cosmological estimates for dark-energy density. So, Beck and Mackey argue, energies at higher frequencies should drop to zero. If that does not happen, they say, we should be looking elsewhere to explain dark energy.

Caldwell says the idea has already been disproved through lab studies of the Casimir effect at very high frequencies, in which no cut-off was observed.

Beck counters that previous estimates of vacuum fluctuations are theoretical, but the Josephson-junction approach offers a direct physical measurement. "The experiment would be cheap and easy to do," he says. 